\title{
Cast Aluminum Energy Consumption Model Based on Fuzzy Petri Net
}

\author{
Yong Sun, Peng Jiang and Guangyu Li \\ Beijing Research Institute of Mechanical and Electrical Technology, Beijing 100083, China
}

\begin{abstract}
An aluminum alloy casting process energy consumption model is created, the model to energy activities as the basic activity unit, description of energy consumption by energy conversion device behavior, to fully reflect the constituent elements of the energy system coordinated through the process energy consumption. The model includes analysis and calculation features, to further simulate the dynamic behavior of business analysis and effective assessment process energy consumption situation of enterprises laid a foundation. The production process and discrete event and time factor (such as equipment failure and maintenance, production instruction scheduling, etc.), so the aluminum alloy casting production system in energy consumption analysis has dual characteristics of continuity and discreteness. According to the above problem, we based on the enterprise energy consumption process, on the basis of the analysis of process energy consumption characteristics, based on Fuzzy Petri net (Fuzzy Petri Nets) method of aluminiums alloy smelting process energy consumption model and the model with energy consumption activities of the basic activity unit, described, through the energy conversion of the energy consumption of energy, fully reflects the energy consumption system component synergy to complete the process of energy consumption.
\end{abstract}

Keywords-fuzzy petri nets; aluminium alloy smelting; energy consumption

\section{INTRODUCTION}

The current research about enterprise energy consumption model is mostly based on production facilities or units for the individual production process, using the mechanism of modeling, identification modeling method to establish mathematical models. These models are produced only from the local point of view emphasize the optimization of production equipment or individual production units, while ignoring the overall production process. Enterprise energy system model is a process model for energy, power equipment and other multi-level model integration model contains. Among them, the energy consumption of the process model should reflect the complete energy system components factor synergistic activity process energy consumption is the core energy system model. Therefore, the establishment of enterprise energy consumption process model, fully reflect the situation of the production process can be expendable and influencing factors, it is to analyze and optimize the energy consumption of the system foundation.

Aluminum casting is a typical process-based industries, in its operation in the dynamic process is always accompanied by the kinetic energy flow and material flow, dynamic, sets, relevance feedback, randomness and other features. In addition, the aluminum casting process, the production process has the characteristics of multi-step, each step accompanied by continuous consumption of materials and energy. At the same time, the production process but also by discrete events and time factors (such as equipment failures, maintenance, production instruction scheduling, etc.) impact, so aluminum casting production systems continuity and discrete dual characteristics in the analysis of energy consumption.

\section{DEFINE THE MODEL}

Definition of fuzzy Petri net energy flow model a six-tuple: (Fuzzy Petri Nets) FPN $=(\mathrm{P}, \mathrm{T}, \mathrm{A}, \mathrm{I}, \mathrm{O}, \mathrm{M0}(\mathrm{p}), \Sigma)$.

1)For $t \in T, M[t>$ works with :

$\forall p \in t: M(p) \geq W(p, t)$

$\forall p \in t^{r}-{ }^{r} t: M(p)+W(t, p) \leq R(P)$

$\forall p \in \mathrm{t}^{r} \cap{ }^{r} \mathrm{t}: \mathrm{M}(\mathrm{p})+W(\mathrm{t}, \mathrm{p})-W(\mathrm{p}, \mathrm{t}) \leq \mathrm{R}(\mathrm{P})$

In which, $\mathrm{t}$ ', ' $\mathrm{t}$ are sets before and after $\mathrm{t}$.

2)If $M\left[t>M^{\prime}\right.$, and $\forall p \in P$ :

$$
\mathrm{M}^{\prime}=\left\{\begin{array}{c}
M(p)-W(p, t), \text { if } \mathrm{P} \in{ }^{\prime} \mathrm{t}-\mathrm{t}^{\prime} \\
M(p)+W(t, p), \text { if } \mathrm{P} \in \mathrm{t}^{\prime}-{ }^{\prime} \mathrm{t} \\
M(p)+W(t, p)-W(p, t), \text { if } \mathrm{P} \in \mathrm{t}^{\prime} \cap{ }^{\prime} \mathrm{t} \\
M(p), \text { else }
\end{array}\right.
$$

1 and 2 showed the formal definition of Petri net.

Among them, place set $\mathrm{P}=P_{\mathrm{o}} \cup P_{\mathrm{a}}=\left\{P_{\mathrm{a}}, P_{\mathrm{g}^{\prime}= \pm}, P_{\mathrm{n}}\right\}$,(n $\geqq 0$ ) is finite set of fuzzy place, by a continuous set of fuzzy sets libraries Pd and discrete fuzzy libraries Pc. Various wherein the continuous fuzzy sets Pc place for material and energy place set representing various energy consumption of production systems (including the consumption of natural gas, electricity, furnace cooling and waste heat, etc.), that the energy flow, or on behalf of the production system input raw materials, auxiliary materials, intermediate products and finished products (including scrap material, copper meters, finished aluminum, 
etc.) and individual equipment, the output buffer (step on a molten aluminum produced), etc., specific performance stream. Discrete Fuzzy place Pd on behalf of the production system status information and control information in the production process, such as the actual production process, start-stop control, process completion status of various monomers set of equipment, etc., to display the actual operational status of the equipment group, Specific performance information flow.

Transition Set $\mathrm{T}=T_{\mathfrak{e}} \cup T_{\mathbb{R}}=\{\mathrm{T} 1, \mathrm{~T} 2, \cdots, \operatorname{Tn}\},(\mathrm{n} \geqq 0)$ is a finite set of fuzzy change. $\mathrm{P} \cap \mathrm{T}=\emptyset, \mathrm{P}$ sets and $\mathrm{T}$ disjoint sets. Transition set $\mathrm{T}$ is decomposed into a continuous transition set $\mathrm{Tc}$ and discrete transition set $\mathrm{Td}$. Change the active set Tc continuous energy on behalf of the system. As well as information related to each discrete set of discrete Td reflect transition in the production process systems interconversion identify discrete and continuous identification, which can be used to token state of the system transition, such as the end of a procedure

$\mathrm{P}$ model to fuzzy fuzzy place transition $\mathrm{T}$, and $\mathrm{T}$ transition to fuzzy fuzzy place $\mathrm{P}$ directed arcs connecting relationship and connection strength, as shown by the association forward matrix (denoted by $\mathrm{P}$ to fuzzy fuzzy place Transition $\mathrm{T}$ directed arc) and backward correlation matrix (represented by fuzzy fuzzy Transition to place T-P directed arcs) :

TABLE I. FORWARD CORRELATION MATRIX

\begin{tabular}{|l|l|l|l|l|l|l|l|l|}
\hline & T1 & T2 & T3 & T4 & T5 & T6 & T7 & T8 \\
\hline P1 & $\alpha_{011}$ & 0 & 0 & 0 & 0 & 0 & 0 & 0 \\
\hline P2 & $\alpha_{021}$ & 0 & 0 & 0 & 0 & 0 & 0 & 0 \\
\hline P3 & 0 & $\alpha_{032}$ & 0 & 0 & 0 & 0 & 0 & 0 \\
\hline P4 & 0 & 1 & 0 & 0 & 0 & 0 & 0 & 0 \\
\hline P5 & 0 & 0 & $\alpha_{053}$ & 0 & 0 & 0 & 0 & 0 \\
\hline P6 & 0 & 0 & $\alpha_{063}$ & 0 & 0 & 0 & 0 & 0 \\
\hline P7 & 0 & 0 & $\alpha_{073}$ & 0 & 0 & 0 & 0 & 0 \\
\hline P8 & 0 & 0 & 0 & $\alpha_{084}$ & 0 & 0 & 0 & 0 \\
\hline P9 & 0 & 0 & 0 & 1 & 0 & 0 & 0 & 0 \\
\hline P10 & 0 & 0 & 0 & 0 & $\alpha_{105}$ & 0 & 0 & 0 \\
\hline P11 & 0 & 0 & 0 & 0 & $\alpha_{115}$ & 0 & 0 & 0 \\
\hline P12 & 0 & 0 & 0 & 0 & 0 & $\alpha_{126}$ & 0 & 0 \\
\hline P13 & 0 & 0 & 0 & 0 & 0 & 1 & 0 & 0 \\
\hline P14 & 0 & 0 & 0 & 0 & 0 & 0 & $\alpha_{147}$ & 0 \\
\hline P15 & 0 & 0 & 0 & 0 & 0 & 0 & 0 & $\alpha_{158}$ \\
\hline P16 & 0 & 0 & 0 & 0 & 0 & 0 & 0 & 1 \\
\hline P17 & 0 & 0 & 0 & 0 & 0 & 0 & 0 & 0 \\
\hline P18 & 0 & 0 & 0 & 0 & 0 & 0 & 0 & 0 \\
\hline P19 & 0 & 0 & 0 & 0 & 0 & 0 & 0 & 0 \\
\hline P20 & 0 & 0 & 0 & 0 & 0 & 0 & 0 & 0 \\
\hline P21 & 0 & 0 & 0 & 0 & 0 & 0 & 0 & 0 \\
\hline
\end{tabular}

TABLE II. BACKWARD CORRELATION MATRIX

\begin{tabular}{|l|l|l|l|l|l|l|l|l|}
\hline & $\mathrm{T} 1$ & $\mathrm{~T} 2$ & $\mathrm{~T} 3$ & $\mathrm{~T} 4$ & $\mathrm{~T} 5$ & $\mathrm{~T} 6$ & $\mathrm{~T} 7$ & $\mathrm{~T} 8$ \\
\hline P1 & 0 & 0 & 0 & 0 & 0 & 0 & 0 & 0 \\
\hline P2 & 0 & 0 & 0 & 0 & 0 & 0 & 0 & 0 \\
\hline P3 & $\beta_{031}$ & 0 & 0 & 0 & 0 & 0 & 0 & 0 \\
\hline P4 & 1 & 0 & 0 & 0 & 0 & 0 & 0 & 0 \\
\hline P5 & 0 & $\beta_{052}$ & 0 & 0 & 0 & 0 & 0 & 0 \\
\hline P6 & 0 & 0 & 0 & 0 & 0 & 0 & 0 & 0 \\
\hline P7 & 0 & 0 & 0 & 0 & 0 & 0 & 0 & 0 \\
\hline P8 & 0 & 0 & $\beta_{083}$ & 0 & 0 & 0 & 0 & 0 \\
\hline P9 & 0 & 0 & 1 & 0 & 0 & 0 & 0 & 0 \\
\hline P10 & 0 & 0 & 0 & $\beta_{104}$ & 0 & 0 & 0 & 0 \\
\hline P11 & 0 & 0 & 0 & 0 & 0 & 0 & 0 & 0 \\
\hline P12 & 0 & 0 & 0 & 0 & $\beta_{125}$ & 0 & 0 & 0 \\
\hline P13 & 0 & 0 & 0 & 0 & 1 & 0 & 0 & 0 \\
\hline P14 & 0 & 0 & 0 & 0 & 0 & $\beta_{146}$ & 0 & 0 \\
\hline P15 & 0 & 0 & 0 & 0 & 0 & 0 & $\beta_{157}$ & 0 \\
\hline P16 & 0 & 0 & 0 & 0 & 0 & 0 & 1 & 0 \\
\hline P17 & 0 & 0 & 0 & 0 & 0 & 0 & 0 & $\beta_{178}$ \\
\hline P18 & $\beta_{181}$ & 0 & 0 & 0 & 0 & 0 & 0 & 0 \\
\hline P19 & 0 & 0 & $\beta_{193}$ & 0 & 0 & 0 & 0 & 0 \\
\hline P20 & 0 & 0 & 0 & 0 & $\beta_{205}$ & 0 & 0 & 0 \\
\hline P21 & 0 & 0 & 0 & 0 & 0 & 0 & $\beta_{217}$ & 0 \\
\hline
\end{tabular}

III. DYNAMIC PROCESS OF THE ALUMINUM CASTING PRODUCTION PROCESS

Related constraints of melting processes is

$$
\begin{gathered}
\tau_{1}=0, \phi 1=(\mathrm{I}(\Pi 1, \mathrm{~T} 1)>0) \cap(\mathrm{I}(\Pi 2, \mathrm{~T} 1)>0), \\
\mathrm{q} 1:\left\{\begin{array}{c}
O(P 3, T 1)=I(P 1, T 1) \\
v(P 1)=-\frac{1}{d_{2}} \\
M 2(h)=M 2(0)+\int_{\Omega}^{h_{2}} v \cdot d s,
\end{array}\right.
\end{gathered}
$$

The energy consumption of this stage of the process:

$$
\mathrm{E} 1=\frac{\mathrm{drg}(\mathrm{n})}{\mathrm{dt}} \cdot \mathrm{E}_{\mathrm{ng}} \cdot \mathrm{d2}
$$

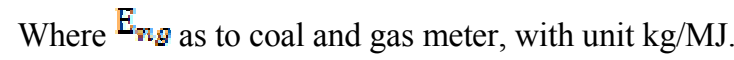

The melting process ends, with its related constraints:

$$
\tau_{2}=0, \phi 2=(\mathrm{I}(\Pi 3, \mathrm{~T} 2)>0) \wedge\left(\mathrm{M} 4\left(d_{z}\right)==1\right)
$$

T2 excitation. 
Related constraints of the alloy process is

$$
\begin{aligned}
& \tau 3=0, \phi 3=(\mathrm{I}(\Pi 5, \mathrm{~T} 3)>0) \cap(\mathrm{I}(\Pi 6, \mathrm{~T} 3)>0 \cap(\mathrm{I}(\Pi 7, \mathrm{~T} 3)>0), \\
& \text { q3: } \\
& \left\{\begin{array}{c}
O(P Q, T 3)=I(P 5, T 3)+I(P 6, T 3) \\
M T(h)=M 7(0)+\int_{Q}^{h} \frac{M \pi(h)}{\pi t} \cdot d s \\
\frac{a n(a)}{d t}=x M / t t / h
\end{array}\right.
\end{aligned}
$$

The energy consumption of this stage of the process:

$$
\mathrm{E} 3=\frac{\mathrm{dur}(\mathrm{t})}{\mathrm{dt}} \cdot \mathrm{E}_{\mathrm{ng}} \cdot \mathrm{dA}
$$

The alloy process ends, with its related constraints:

$$
\tau 4=0, \phi 4=(\mathrm{I}(\Pi 8, \mathrm{~T} 4)>0) \wedge\left(\mathrm{M} 9\left(d_{4}\right)==1\right),
$$

T4 excitation.

\section{VERIFICATION OF THE AVAILABILITY OF THE ENERGY FLOW MODEL}

In HTXG.AC3AM aluminum production operations, for example, according to the working mechanism of production conditions and various equipment under set conditions corresponding parameters, this model simulation. In an actual production records furnace temperature data, compared to aluminum casting production process to identify transition in stream temperature simulation model place, the results showed consistent trend between transition represented by fuzzy place basically reflects the production process.

Actually measured furnace temperature curve of HTXG.AC3AM aluminum production:

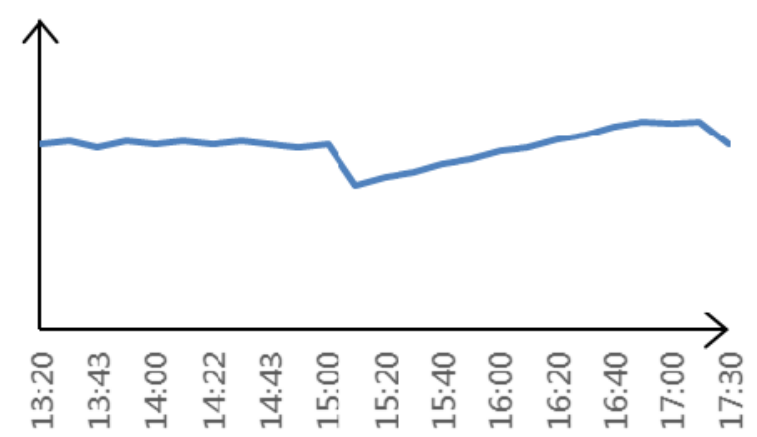

Transition in temperature place tokens:

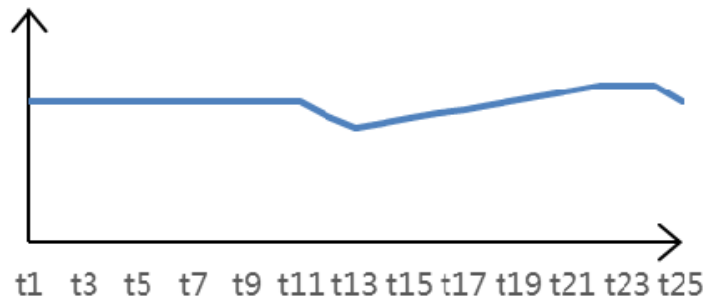

\section{CONCLUSION}

Based on enterprise energy consumption process, in the process of analyzing the characteristics of energy consumption on the basis of fuzzy Petri nets (Fuzzy Petri Nets) way to create aluminum alloy casting process energy consumption model, the model to energy activities as the basic activity unit, description of energy consumption by energy conversion device behavior, to fully reflect the constituent elements of the energy system coordinated through the process energy consumption. The model includes analysis and calculation features, to further simulate the dynamic behavior of business analysis and effective assessment process energy consumption situation of enterprises laid a foundation.

\section{REFERENCES}

[1] YANG Youqi, XIANG Shuguang. Chemical process simulation and optimization [ M ] . Beijing: Chemical IndustryPress,2006: 92-108( in Chinese) .

[2] State Council of China, Made in China 2025 Planning Outline. 2015.

[3] MA Fumin, WANG Jian. Fuzzy Petri nets model oriented to enterprise energy consumption process [ J ] . Computer Integrated Manufacturing Systems, 2007, 13 ( 9) : 1679-1685.

[4] SID HUTS, AOZ. On-line evaluation of capacity and energy losses in power transmission systems by using artificial neural networks [ $\mathrm{J}]$. IEEE Transactions on Power Delivery, 1995,10( 4) : 1913-1919.

[5] GHOMRIL, ALLAH . Modeling and analysis using hybrid Petri nets [ J ] . Nonlinear Analysis: Hybrid Systems, 2007, 1( 2) : 141-153. 